\title{
IOT Based Prepaid Purified Water Supply And Monitoring System For Housing Society
}

\author{
${ }^{1}$ Nikhil Patil, ${ }^{2}$ Ajinkya Pawar, ${ }^{3}$ Omkar Rane, ${ }^{4}$ Dr. Vinayak Shinde \\ ${ }^{1,2,3}$ Student, ${ }^{4}$ Head and Associate Professor, Dept of Computer Engineering, Shree L.R Tiwari \\ College of Engineering, Thane, India, ${ }^{1}$ nikhil.patil4599@gmail.com, ${ }^{2}$ ajinkyapawar886@gmail.com, \\ 3 omkarrane23041999@gmail.com, 4vdshinde@gmail.com
}

\begin{abstract}
There are so many diseases which are caused because of unfiltered water. In the market many products are available for filtration of water, but all that product needs a huge amount of cost for maintenance and installation process. So there is the need for filtration of water at low cost and efficiently. For that there will be an automated system which takes payment in advance and then supplies the water according to the purchased plan,which is based on the user's needs. Hence the purpose of this project is to develop a system which is based on prepaid payment for water supply and monitoring water supply system for housing society. Water supply is measured by using flow control to control the flow of water according to the usage of the user. By doing this we can decrease the rate of human errors. All payment transactions are recorded in the system for further reference. This System evolves Automatically because it detects the recharge details from the message. After that it will give supply to that user according to their recharge details. This System is fully automated which can overcome the problems of improper bill creation, delay in bill availability and delay in payment process. Also system notify users for upcoming recharge and for water consumption.
\end{abstract}

Keywords —Prepaid water supply, Monitoring water supply, Automated System, AWS S3, AWS EC2, AWS IOT-Core, AWS Dynamodb, AWS SNS, Paytm Payment Gateway

\section{INTRODUCTION}

From an article of yourstory which says that Unlike electricity, water does not have an alternative source. Till date, no viable solution has been found for fresh water production. However, time and again, communities have invested heavily in conserving water. Rain water harvesting, well rejuvenation, and ground water recharge practices have gained prominence over the past few decades. Water conservation must become a duty for every human being, else global water wars are not far away [1].

According to NASA, One-third of Earth's largest groundwater basins are being rapidly depleted by human consumption. Significant segments of Earth's population are consuming groundwater quickly without knowing when it might run out. But only water supply is not a problem because there were many diseases caused because of unfiltered water. Hence there will be some solution which will supply purified water. For this our proposed system will work on a prepaid mechanism, where after payment of the user this system will purify the water supply [12].

\section{Problem Statement}

"To develop a system based on a prepaid mechanism for purified water supply and monitoring system for housing society using Internet of Things(IOT)".

In that purified water supply is given to homes by using a flow control sensor where measurement should be taken according to usage. Flow control sensor will give measurement as an input from where we can on/off solenoid. Where all that recharge details made by the user will be saved in the database for record purposes.

\section{LITERATURE SURVEY}

Paper [2], describes System for Prepaid Smart card System for Water Supply, for minimum loss of water ,in this system has been implemented using microcontroller IC with the help of serial data transfer Inter-Integrated Circuit protocol. The system is designed in such a way that memory IC has been used as a smart card on which recharge is done. Our system is not based on smart cards. Paper [3], describes the System for Automatic Water Billing System based on Android Application, this system passes readings from microcontroller only when the valves are closed. bill and usage can be viewed on android app where payment of bill is done using an online payment method. 
Paper [4],describes System IOT Based Automated Water Billing System (AWBS),this system uses digital water flow rate sensor instead of analog meter ,based on usage bill is sent to user through SMS at the end of every month, by gathering all information about water use it will shown usage in the form of graphs and charts using cloud platforms "thingspeak" and "pushing box". where this proposed system is implemented with "AWS" cloud platform. Paper [5], describes the System of Smart Water Monitoring System using IoT, this system also monitors water supply with additional features like Ultrasonic Sensor used to measure water level on the tank. The other parameters like $\mathrm{pH}$, TDS, and Turbidity of the water can be calculated using different corresponding sensors. Paper [6], describes the IOT Based Smart water Supply Management System, System is to monitor water level and measure water supply. Where in this system users need to pay according to their daily usage, hence the recharge system is based on a postpaid mechanism. where our proposed system based on prepaid mechanism.

\section{Proposed System}

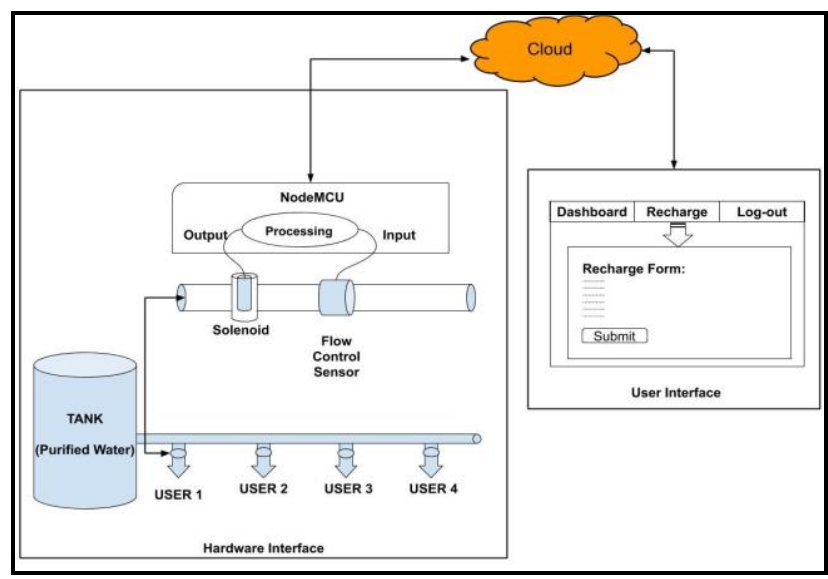

Figure.1: Proposed Diagram for the system

Figure 1 above depicts the system of the project. It consists of three modules which are Hardware interface ,user interface and cloud [10][11].

Full system works on the internet. Users will interact with the system using a application. From that application user will first need to make their account after that only it can use that application. When a user wants water then, the user will first pay according to the selected plan. After that users can get their purified water. Payment is done by using paytm API for that user needs to have a paytm account. When recharge is done then this system detects it according to user requirement it will set their counter to that limit i.e if the user wants 5 liter of water supply then this system measures water flow and count till it reaches 5 liter. where according to user consumption it will decrease the counter [7].This monitoring and measurement of the system is done by using Microcontroller which is NodeMCU [8]. It will send the instruction via the internet from that system will track user consumption of water usage on their daily bases. Through NodeMCU, flow control and solenoid sensor is connected which will be input and output for the system [8].

Which means flow control sensor work as an input device where from that system give feedback for closing water supply to solenoid valve which is nothing but an output device. Both User interface and hardware interface are connected by the help of cloud [11].

\section{SySTEM REQUIREMENTS}

\section{A. Hardware Requirements}

- Node MCU-microcontroller

- Solenoid valve-for controlling water supply

- flow control sensor-for measuring water supply

- 4 channel $5 \mathrm{v}$ relay module-supplying $12 \mathrm{~V}$ power supply for solenoid valve

\section{B. Software Requirements}

- Spring Source Tool -for backend coding

- Visual Studio Code-for frontend coding

- Wamp Server -for Mysql database

- PostMan -for testing restful API's

- AWS EC2-for virtual server

- AWS Dynamodb(NO Sql)-for sensor data

- AWS SNS-for sending notification

- AWS S3-for storing website storage

- AWS IOT-Core-for register iot thing in cloud

- AWS RDS-for storing MySql database on cloud

\section{System DESIGN}

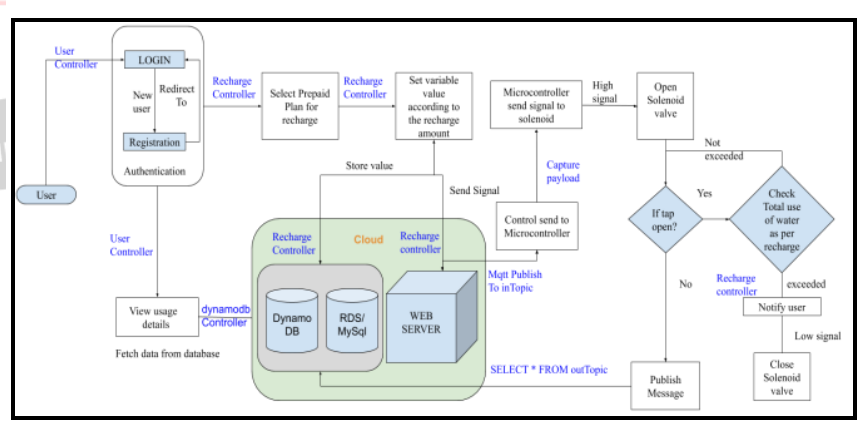

Figure.2: Block Diagram for the system

Above Figure 2, shows the block diagram of the system. The system consists of a hardware connection and software application with Internet connection. Initially users will login to the system, if not register it will register from sign up page with all details like age, email, family members etc. After that user will have access to the system, Further if the user wants to buy water then he/she must select a prepaid plan according to need and make payment using paytm method. After Successful recharge system will supply water according to the recharge plan . Using web service form cloud we communicate to microcontroller board using MQTT protocol Publish/Subscribe method.. 
Microcontrollers will publish topics to solenoid and flow control. where if payment is done then from internet signal sent to microcontroller and from microcontroller signal will be sent to solenoid wall, so that system will start water supply. If tap is open and if consuming water is less than total water specified in recharge plan then it stores that count in database which is also on cloud and retain valve open .System will take measurement by using a flow control sensor which measures the amount of water supplied to the user. Flow Control sensor works on hall effect. Once the user crosses the limit of the recharge plan then the microcontroller sends a signal for closing the valve.System will also track previous records which will help users to view their past usage [10]. Tracking will also be useful for medical analysis of users which will show user medical condition because of consuming less amount of water.

\section{METHODOLOGY}

First, making an application which will interact with users should be made by using Spring boot technology which is nothing but an java framework and angular as a front end. While form backend which is spring boot we create restful api's, then by using angular framework we are consuming them using http protocol.

After that in the second phase, we establish mqtt connection with NodeMCU and AWS cloud. where on AWS we are using IOT core service which is useful for mqtt connection, using that we can subscribe topics, where we subscribe two topics one is inTopic and other is outTopic,inTopic is useful for sending instruction from IOT core(AWS cloud) to NodeMCU, where outTopic is useful for sending data from NodeMCU to IOT core(AWS cloud) ,both are shown in figure 3(a) and figure 3(b) [9].

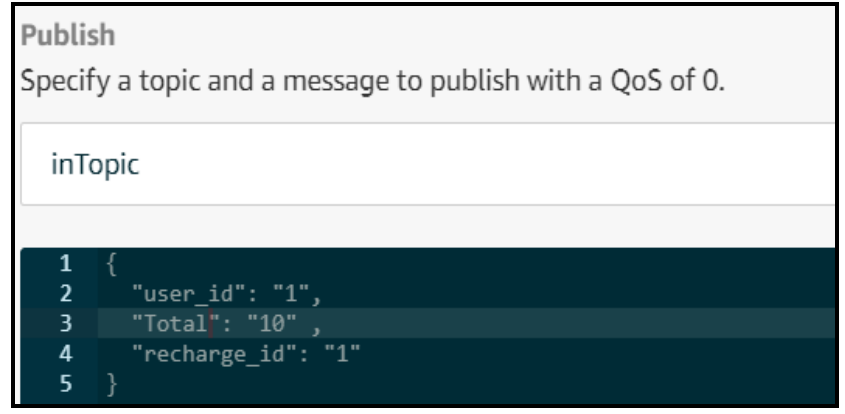

Figure.3(a): IOT Core service inTopic

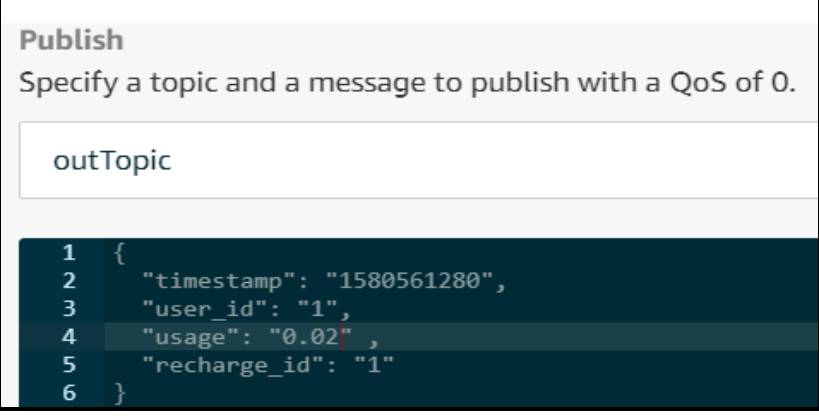

Figure.3(b): IOT Core service outTopic
After selecting a plan of recharge amount we use Paytm payment gateway for payment purposes. hence we integrate Paytm Api with our spring boot app which is mention in figure 4. paytm api needed server parameters which we will mention using the backend.

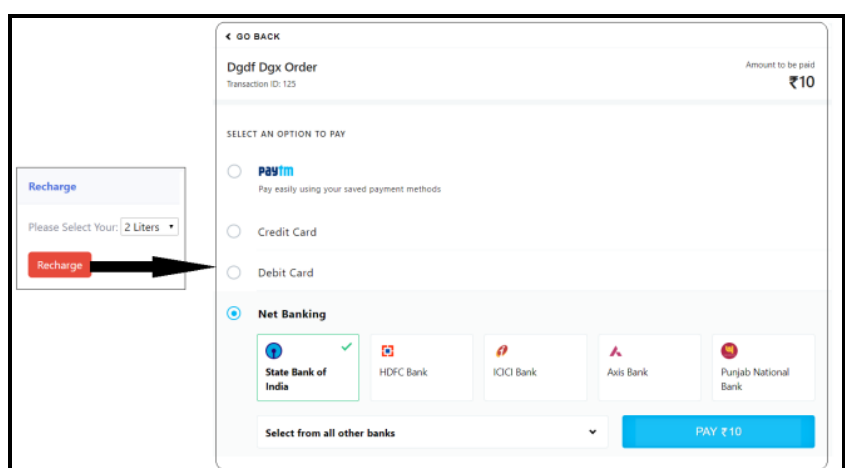

Figure.4: Paytm Api gateway integration

But for storing outTopic JSON data we are using DynamoDB which is also an AWS service [9]. DynamoDB is nothing but a NoSQL database. While storing data on DynamoDB we set one partition key which is timestamp and other fields are user_id, recharge_id with their usage amount in liters this is shown in figure 5 .

\begin{tabular}{|c|c|c|c|c|}
\hline \multicolumn{2}{|c|}{ Create item } & \multicolumn{3}{|c|}{ Actions $\vee$} \\
\hline \multicolumn{5}{|c|}{ Scan: [Table] usage: timestamp $\vee$} \\
\hline & timestamp $\mathbf{i}$ & recharge_id & usage & user_id \\
\hline 0 & 1580561280 & 99 & 0.053567 & 75 \\
\hline 0 & 1580561281 & 99 & 0.310738 & 75 \\
\hline 0 & 1580561282 & 99 & 0.350511 & 75 \\
\hline$\square$ & 1580561322 & 99 & 0.384934 & 75 \\
\hline 0 & 1580561323 & 99 & $0.553809 \square$ & 75 \\
\hline
\end{tabular}

Figure.5: DynamoDB table data

We use this usage data from DynamoDb to display on user interactive application in a form of chart and for that use chart.js and using this we display usage for selected time intervals which is shown in figure 6 .

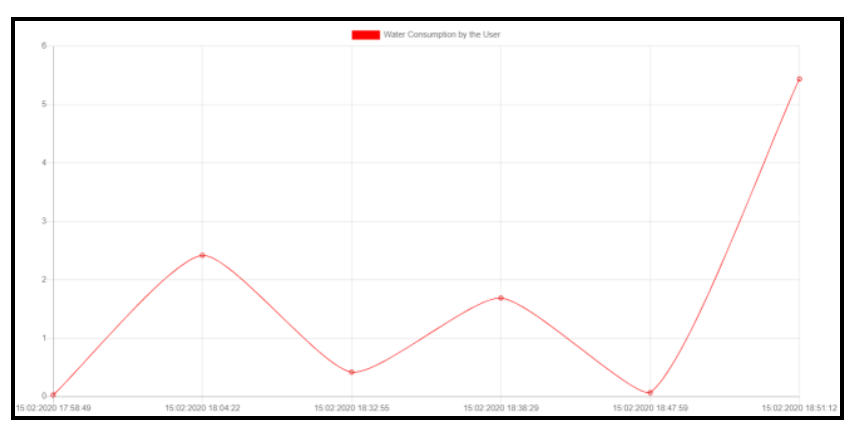

Figure.6: Water usage as per 5 minutes

For alert notification We use SNS service from AWS. For security of our application we suses both server side and UI/UX side security. For that we use the JWT form server side for authentication of every user. For deployment 
of our application we use EC2, S3 and RDS services form AWS.

\section{RESUlts}

Our project is a combination of Hardware components and user interactive application. Some of the snapshots of result are listed below. Where figure 7 describes our hardware connectivity.

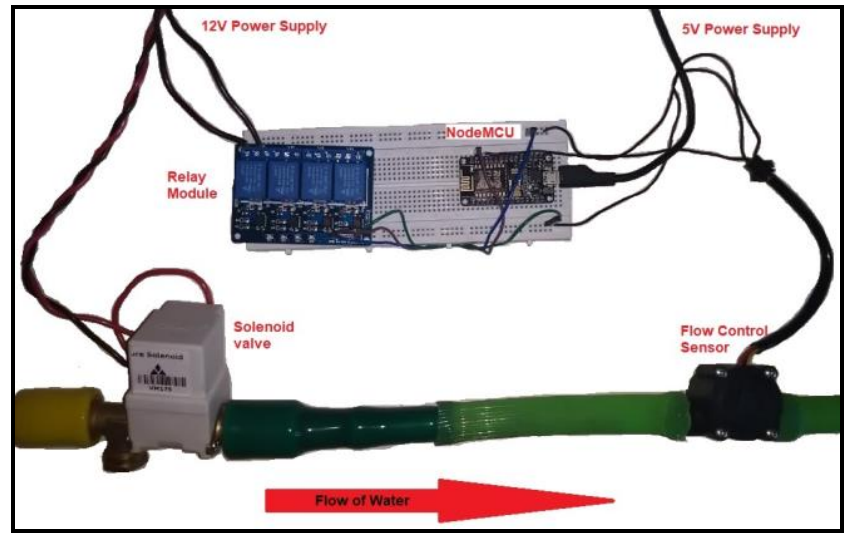

Figure.7: Hardware components connections

Figure 8 Shows the Registration form for the user want to use our service. At the time of registration, user have to enter some basic and required details like name, email, phone number, age and Total number of members in the house and their details. It also has a consent for admin for accessing the data.

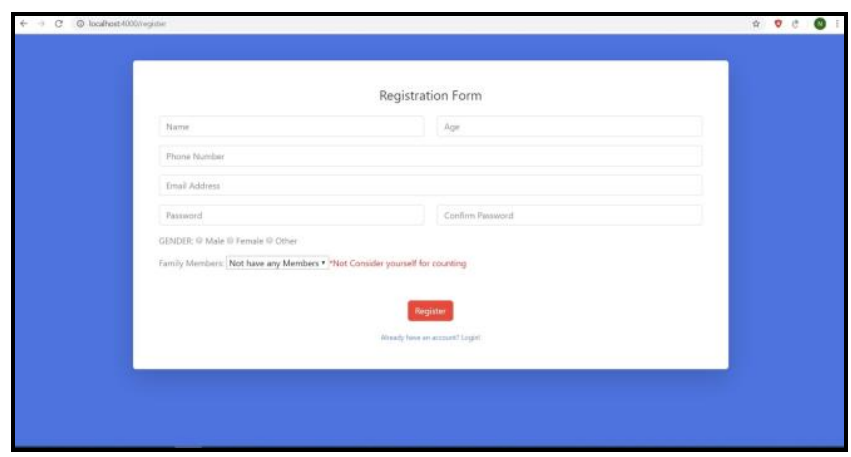

Figure.8: Registration Form

Figure 9 Show the login form for the users that have registered already and for login purpose users have to enter their email address and password. This page also has a link to registration purpose if the user is not registered.

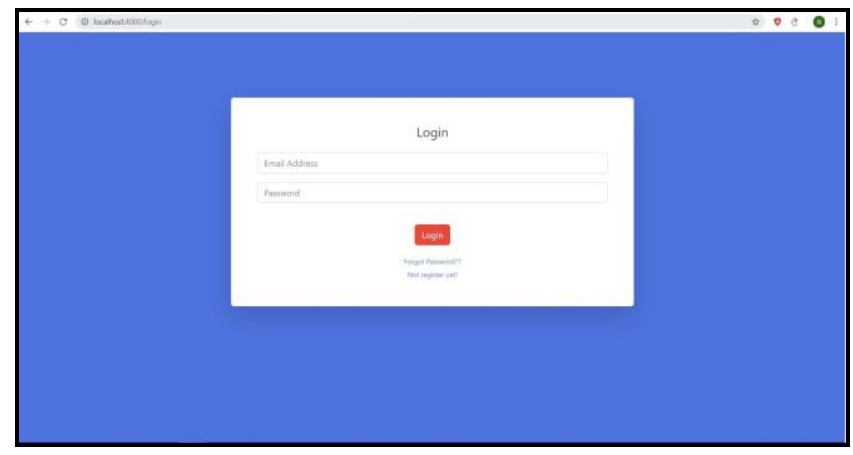

Figure.9: Login page
If any user forgets password and then he will not be able to login to the application so this page is for resetting password with new password created by user this can be done by reset password page shown in figure 10 .

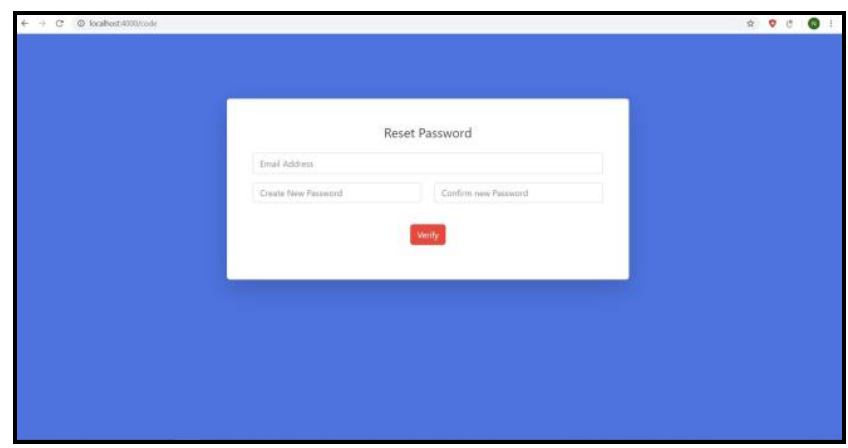

Figure.10: Reset Password

After successful login user will redirect to dashboard and it contains various links to various operations like Recharge, Usage, Recharge History and Logout functionalities. Also dashboard gives the overall information about the project and display the pie chart of the user health status(HealthyUnhealthy) show in figure 11.

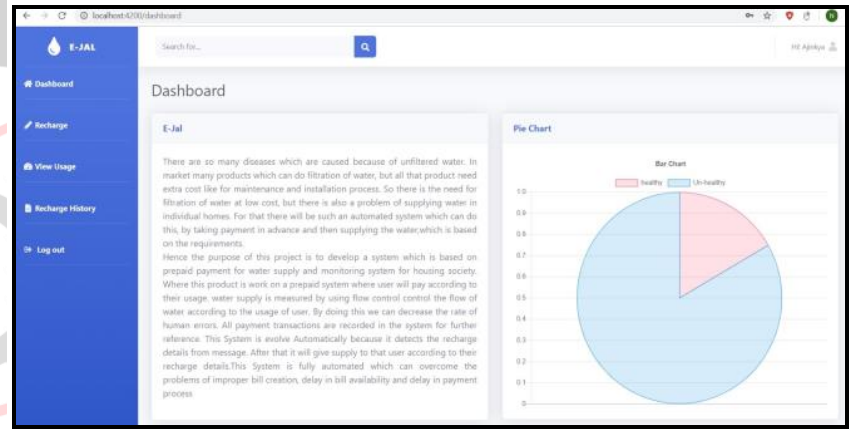

Figure.11: Dashboard page.

Figure 12 shows the functionality of view user page ,in this page use can view its own details provided at the time of registration and it displays the users and its members information's like name, age, phone number etc.

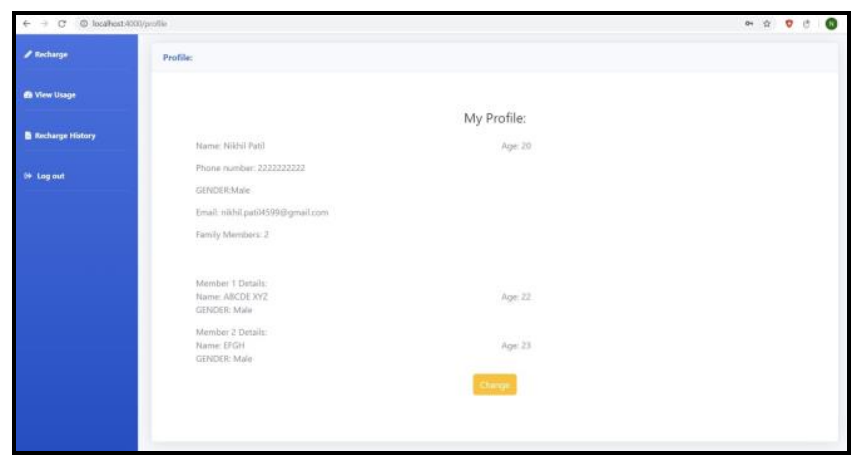

Figure.12: User Profile

Figure 13 show the functionality of update user details ,in this page user has option so that he can update its own details provided at the time of registration and he can update its own details or its members information's like name, age, phone number etc. 


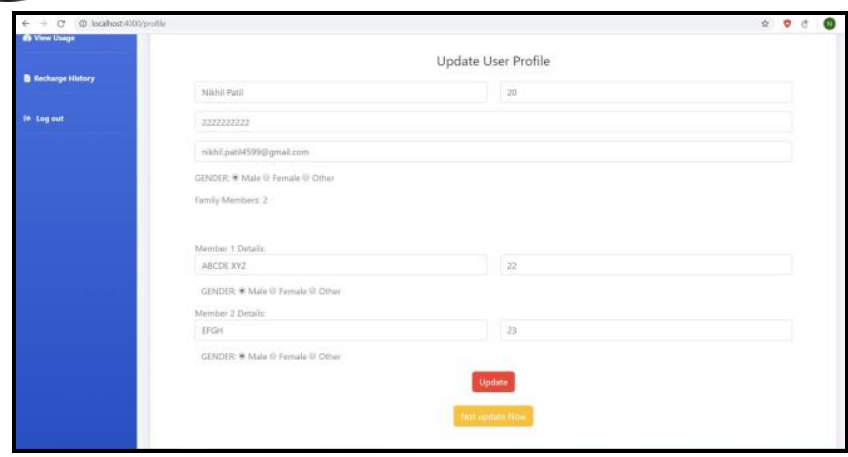

Figure.13: Update User Profile

Figure 14 shows the functionality of selection of recharge plan. It will allow users to select various plans like 2,5,10 liters and if the user is done recharge then the recharge button is disabled to avoid extra recharge and it will get automatically activated once the current plan expires.

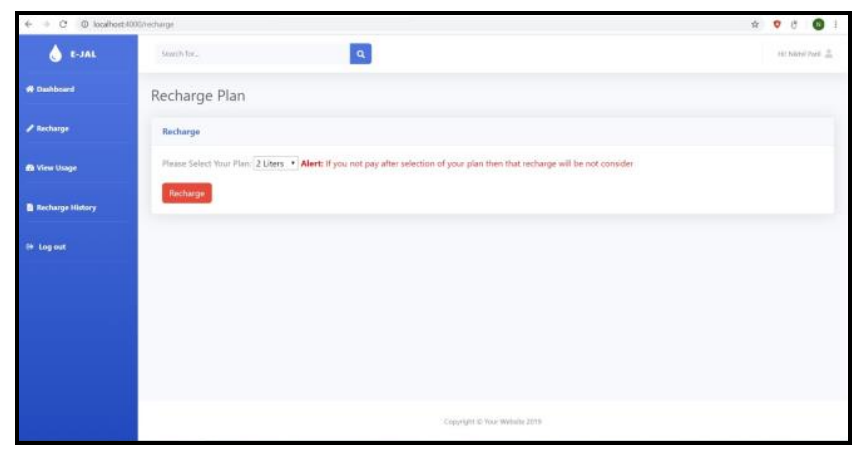

Figure.14: Select Recharge Plan

Figure 15 shows the Line chart of Current Water consumption of the user, it provides the options to select the various time period so that user can view the usage of that selected time period and it also display the timestamp.

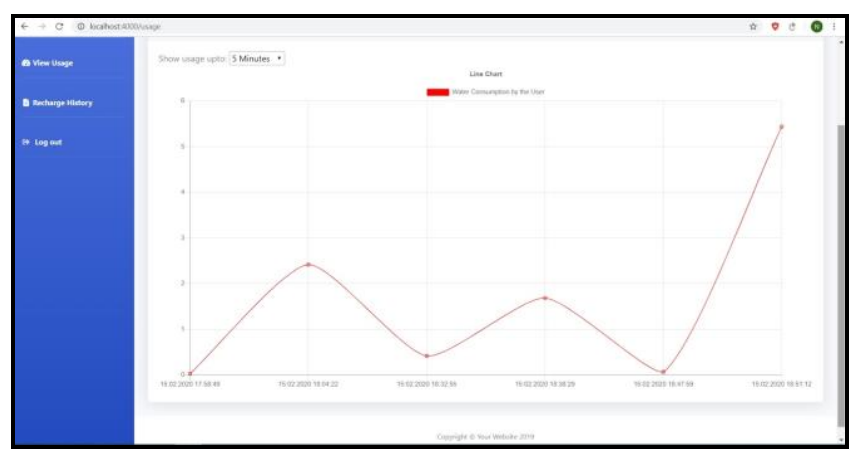

Figure.15: Usage graph page

Fig 16 Shows the history of the all the recharges done by the user. It show the plan, date on which recharge is done ,status and the bill amount of the recharge also it provides the option by which we can view the each recharge details in more detail manner.

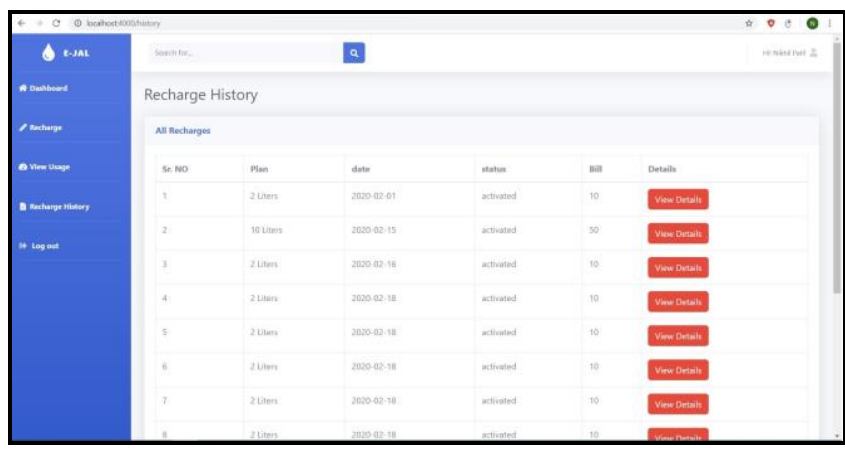

Figure.16: Recharge History page

Figure 17 shows the selected recharge detail and it shows the response which is received after the successful recharge and it has various parameters like transaction id ,order id, txn status, bank details etc.

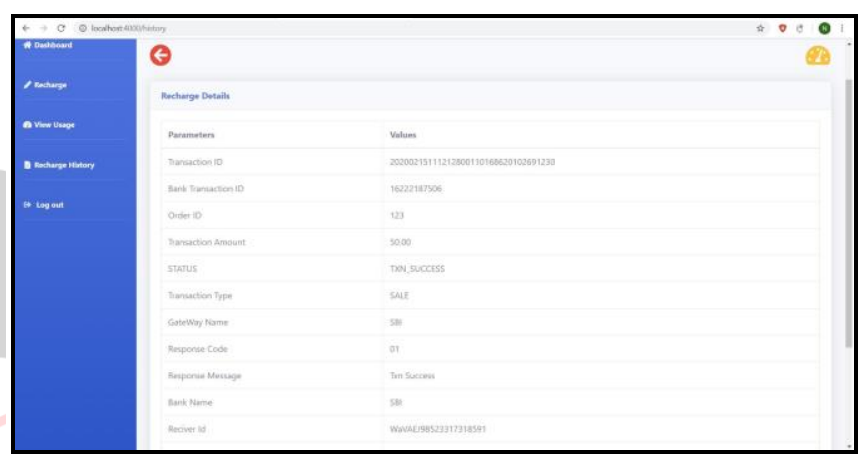

Figure.17: Recharge details

Figure 18 shows the Line chart of past water consumption of the user, it provides the options to select the various time periods like 5,10,30 minutes so that the user can view the usage of that selected time period and it also displays the timestamp.

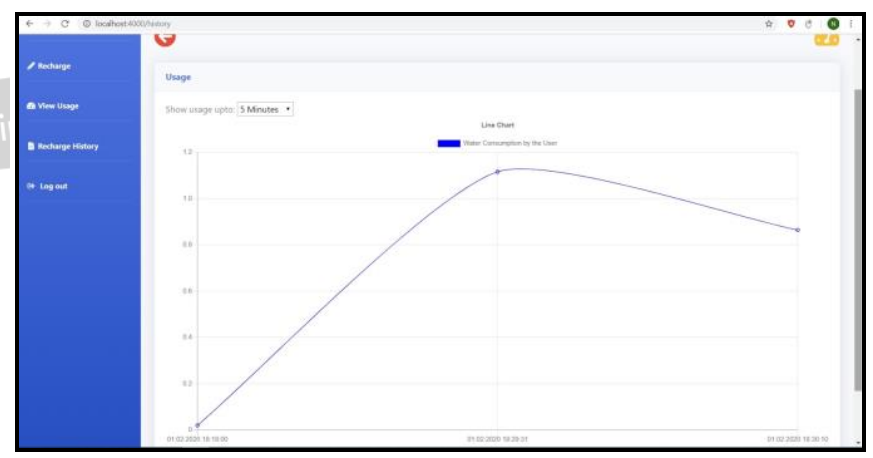

Figure.18: Graph of past recharge

Some of the following listed figure shows the mobile application which has almost all the features that are available in web applications. All this figures are shown in figure 19.1,19.2 and 19.3. 


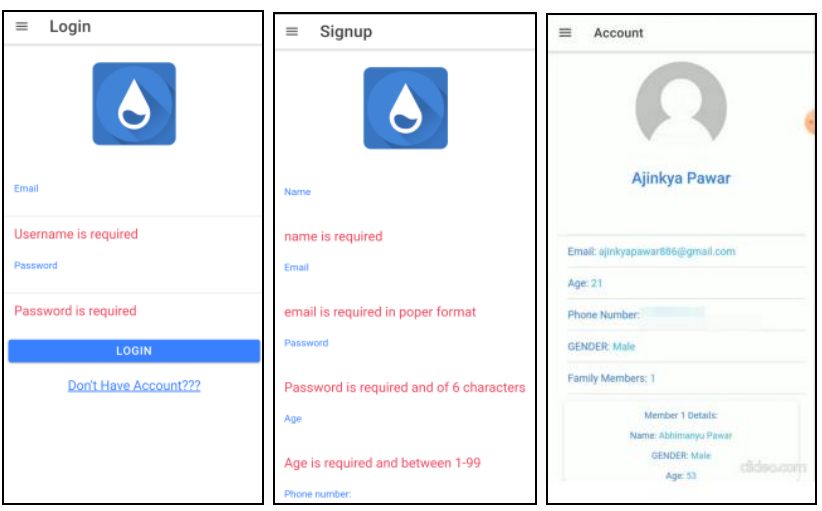

Figure.19.1: Login, Registration and View profile Page.

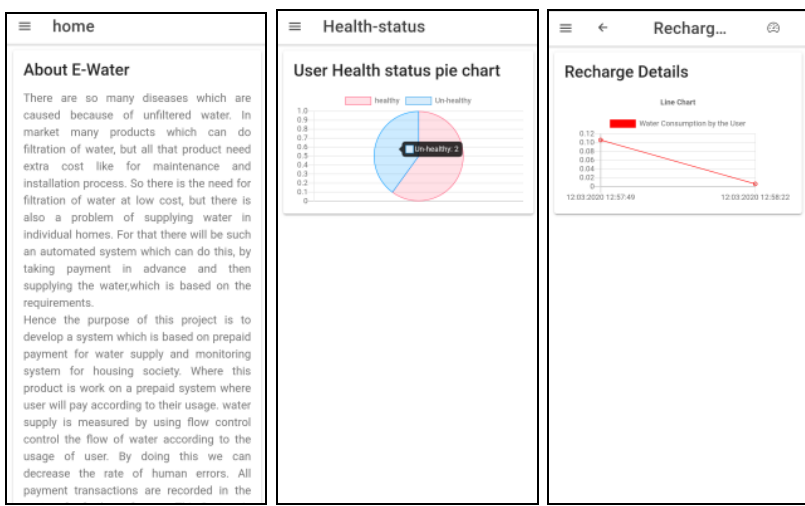

Figure.19.2: Dashboard, Health Report and Graph.

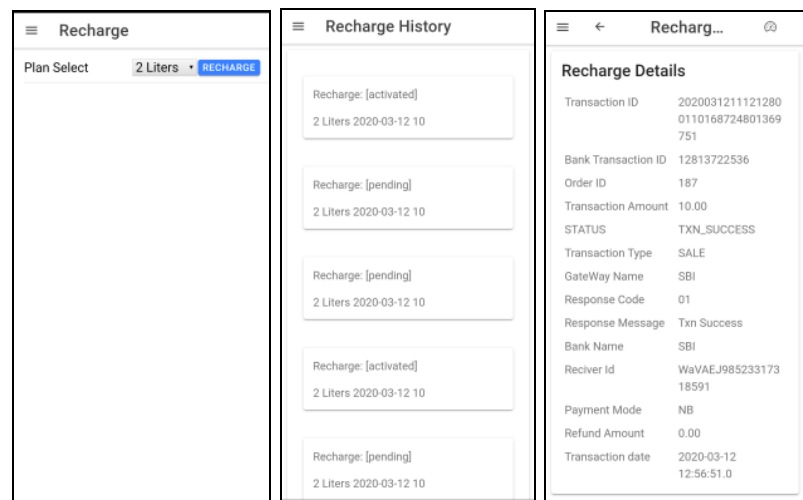

Figure.19.3: Recharge, Recharge history and Recharge details

Figure 20 shows the notification received in the users mobile phone ,notification is received when the recharge plan is expired and another notification is received every day which shows the health status of the user according to water consumption.

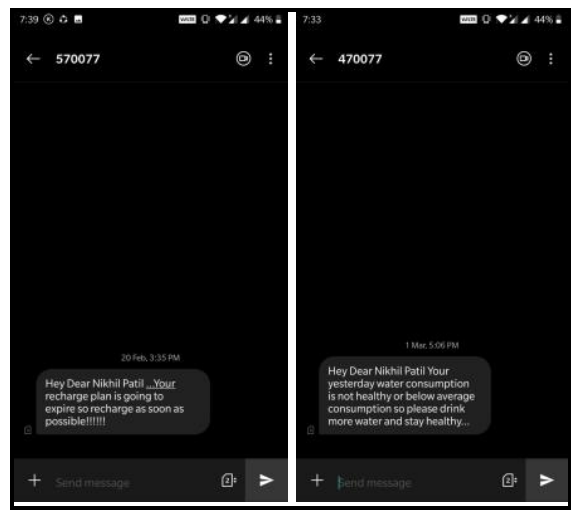

Figure.20: Notification of Recharge Expire and Unhealthy Report of 24

Figure 21 shows the prototype/model of our system which represents the structure of housing society (building) and water tank and pipe connection of the houses.

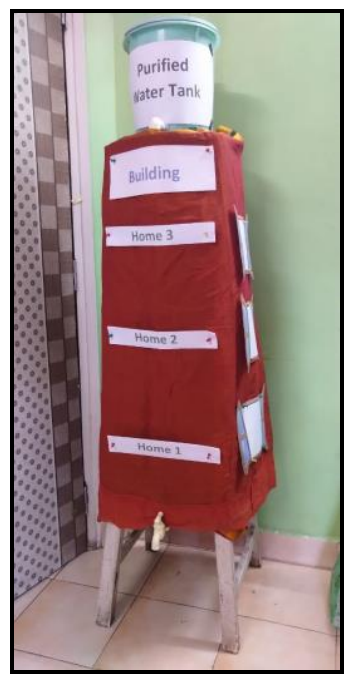

Figure.21: Final Model of our system

\section{CONClusion ANd Future SCOPE}

Our proposed system can be implemented using dedicated hardware which are mentioned in system requirements which are also cost efficient. As we mention that this system tracks usage details of any user so that we can give notification alerts according to their usage. If a user uses less water per day then we give notification alerts so users can be aware about their health. We are trying to implement this proposed system within a housing society so we can also give health reports to the health department so that it will also be useful for avoiding health issues.

\section{REFERENCES}

[1] J. Gouthaman, R. Bharathwajanprabhu and A. Srikanth, "Automated urban drinking water supply control and water theft identification system," IEEE Technology Students' Symposium, Kharagpur, pp. 87-91, 2011. 
[2] G. P. Jain, R. G. Jain and P. S. Bangale, "Prepaid Smart Card System for Water Supply," IOSR Journal of Electronics and Communication Engineering, vol. 1, no. 1, pp. 1-4, 2015.

[3] R. B. Dhumale, N. D. Thombare and P. M. Bangare, "Automatic Water Billing System based on Android Application," International Research Journal of Engineering and Technology, vol. 5, no. 8, pp. 977982, 2018.

[4] B. P, A. S. N and A. Karan, "IoT Based Automated Water Billing System," International Journal of Engineering Research in Computer Science and Engineering, vol. 4, no. 4, pp. 53-57, 2017.

[5] G. J, C. R. Reddy, P. Meher, S. Shrivastava and G. Kumar, "Smart Water Monitoring System using IoT," International Research Journal of Engineering and Technology, vol. 5, no. 10, pp. 1170-1173, 2018.

[6] K. M. Dhobale, S. P. Gorgile, P. J. Gunjal, K. A. Hirve and P. U. A. Mande, "IOT based Smart Water Supply management," International Journal of Advanced Research in Computer and Communication Engineering, vol. 6, no. 1, pp. 297 299, 2017.

[7] K. S. S and A. G. N, "An IOT based Water Supply Monitoring and Controlling System," International Research Journal of Engineering and Technology, vol. 05, no. 02, pp. 857-860, 2018.

[8] T. Rahman, T. Ahmed, I. Hasan and M. A. Alam, "Automated Household Water Supply Monitoring \& Billing," in IEEE Xplore Compliant, Bangladesh, 2018, pp. 448-455.

[9] J. Baron, H. Baz, T. Bixler and B. Gaut, AWS Certified Solutions Architect, Canada: John Wiley \& Sons, Inc. Indianapolis, Indiana, 2017, pp. 1-517.

[10] S. Saseendran and V. Nithya, "Automated Water Usage Monitoring System," in IEEE Advanced Technology for Humanity, India, 2016, pp. 99-103.

[11] S. B. Saraf and D. H. Gawali, "IoT based smart irrigation monitoring and controlling system," in 2 nd IEEE International Conference on Recent Trends in Electronics, Information \& Communication Technology (RTEICT), Bangalore, 2017, pp. 815819.

[12] V. Radhakrishnan and W. Wu, "IoT Technology for Smart Water System," in IEEE 20th International Conference on High Performance Computing and Communications; IEEE 16th International Conference on Smart City; IEEE 4th International Conference on Data Science and Systems (HPCC/SmartCity/DSS), Exeter, United Kingdom, 2018, pp. 1491-1496. 